\title{
Oxygen Sensitivity of Intermetallic Compound Surface Comprising Early Transition Metal
}

\author{
Shinya Furukawa*, Genki Nishimura, and Takayuki Komatsu* \\ Course of Chemistry, School of Science, Tokyo Institute of Technology, \\ 2-12-1 Ookayama, Meguro-ku, Tokyo 152-8551, JAPAN
}

(Received April 22, 2016)

\begin{abstract}
Pd-based intermetallic compounds having early transition metals $\left(\mathrm{Pd}_{3} \mathrm{M} ; \mathrm{M}=\mathrm{Nb}, \mathrm{Ti}\right.$, and $\left.\mathrm{Zr}\right)$ prepared by arc melting were characterized by X-ray photoelectron spectroscopy. When the ingot of $\mathrm{Pd}_{3} \mathrm{Zr}$ was ground in $\mathrm{N}_{2}$ with an oxygen concentration below $1 \mathrm{ppb}$, a large part of the surface maintained its original intermetallic state; however, for $\mathrm{Pd}_{3} \mathrm{Nb}$ and $\mathrm{Pd}_{3} \mathrm{Ti}$, their surfaces were completely oxidized even in the $\mathrm{N}_{2}$ atmosphere. This indicates the remarkably high oxygen sensitivity of the intermetallic compounds with early transition metal.
\end{abstract}

Keywords: Intermetallic compound, Early transition metal, Oxygen sensitivity, XPS, Palladium

\section{Introduction}

Intermetallic compounds are stoichiometric compounds of more than one metal element, which typically have specific crystal and electronic structures compared with those of the parent metals ${ }^{1)}$. The use of intermetallic compounds as catalysts has received increasing attention ${ }^{2}$. Intermetallic compounds that are expected to be promising catalysts comprise catalytically active elements, such as later transition metals (e.g., Pt, $\mathrm{Pd}, \mathrm{Rh}, \mathrm{Ni}$, and $\mathrm{Co}$ ). Therefore, the counterpart metal should be an early transition or typical metal that is set apart from the later transition metals in the periodic table. Intermetallic compounds having typical elements have been widely studied and revealed as effective catalytic materials; for example, $\mathrm{Pt}_{3} \mathrm{Sn}$ for alkane dehydrogenation ${ }^{3)}, \mathrm{Pd}_{3} \mathrm{~Pb}$ for methyl methacrylate synthesis ${ }^{4)}$ and amine oxidation ${ }^{5)}$, and PdGa for acetylene semihydrogenation ${ }^{6)}$. On the contrary, intermetallic compounds having early transition metals have rarely been studied as catalysts; hence, they are of great interest for catalytic chemistry.

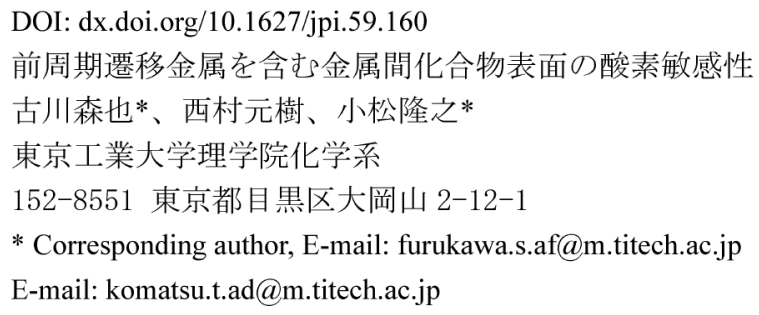

This may be partly due to their surfaces being labile in air. As early transition metals have significantly high ionization tendencies, the surface of their intermetallic compounds seems to easily undergo oxidative decomposition in the presence of oxygen to form oxide phases. Moreover, such oxides are hard to be reduced by gaseous hydrogen at high temperatures (400$800{ }^{\circ} \mathrm{C}$ ), the typical pretreatment for metallic catalysts. Therefore, experimental procedures, including the catalyst preparation and its transfer into a reactor or spectrometer, should be performed under oxygen-free conditions, such as under an ultra-high vacuum or pure inert gas atmosphere.

In this study, we prepared several Pd-based intermetallic compounds having early transition metals $\left(\mathrm{Pd}_{3} \mathrm{M}, \mathrm{M}=\mathrm{Ti}, \mathrm{Zr}\right.$, and $\left.\mathrm{Nb}\right)$ and characterized their surface under an inert atmosphere with ppb levels of oxygen. We report remarkably high oxygen sensitivity of the early transition metals at the surface of the intermetallic compounds.

\section{Experimental Details}

\subsection{Glove Box Conditions}

The inert gas atmosphere was prepared using an UNICO UL-800A glove box equipped with an MF-100 circulating gas purifier (for deoxygenation) and TORAY SD-450 $\mathrm{O}_{2}$ sensor (range: $1 \mathrm{ppb}-100 \%$ ). Dry nitrogen (Taiyo Nippon Sanso, $99.99995 \%$ ) was used as an inert gas. Standard working conditions typically result in an oxygen concentration of 500-600 ppb, 
which may be attributed to the purity of the supplied $\mathrm{N}_{2}$. In this study, ISO A-500HS oxygen absorbers (iron powder) were used to minimize oxygen concentration and air exposure. First, the supplied $\mathrm{N}_{2}$ was purified through oxygen absorbers placed at the $\mathrm{N}_{2}$ inlet. Second, the butyl rubber gloves were overlapped with extra gloves to prevent exposure of the sample to percolated oxygen. Several oxygen absorbers were put between the parent and extra gloves to trap the percolated oxygen. These efforts allowed an oxygen concentration below 1 $\mathrm{ppb}$ (indicated as $0 \mathrm{ppb}$ ) during experimental procedures.

\subsection{Catalyst Preparation}

Pd-based intermetallic compounds $\left(\mathrm{Pd}_{3} \mathrm{M}, \mathrm{M}=\mathrm{Ti}, \mathrm{Zr}\right.$, and $\mathrm{Nb}$ ) were prepared by arc melting of a mixture of Pd (Kojundo Kagaku, $99.9 \%$ ) and the second metal (Soekawa Chemical, $99.9 \%$ ) under an Ar atmosphere using a SANVAC SV-A434 vacuum arc-melting furnace. The resultant ingots were transferred into the glove box and ground into a fine powder. For grinding, the oxidized shell part of the ingot was discarded and the core part was collected as a naked sample. As a control experiment, grinding in air with a subsequent reduction treatment was also conducted. The ingot of the intermetallic compound that was ground in the air was treated by $\mathrm{H}_{2}$ in a separable glass tube connected to a gas circulation system (under $60 \mathrm{kPa} \mathrm{H}$ at $800{ }^{\circ} \mathrm{C}$ for $1 \mathrm{~h})$. The sample in the sealed glass tube was transferred into the glove box without exposure to air.

\subsection{Characterization}

X-ray photoelectron spectra (XPS) of the intermetallic compounds were recorded with an ULVAC PHI 5000 VersaProbe spectrometer. The ground catalyst powder was pressed into a pellet and placed on a sample holder for the spectrometer in the glove box. This sample holder was transferred into the spectrometer without being exposed to air using a portable transfer vessel. Spectra were obtained with an Al K $\alpha$ X-ray source using $C 1 \mathrm{~s}$ as a reference for binding energy. The crystal structures of the prepared intermetallic compounds were examined in the air by powder X-ray diffraction (XRD) with a Rigaku RINT2400 using a $\mathrm{Cu} \mathrm{K} \alpha \mathrm{X}$-ray source.

\section{Results and Discussion}

The powder ground in air was analyzed by X-ray diffraction. For all samples, the desired intermetallic phases $\left(\mathrm{Pd}_{3} \mathrm{Nb}, \mathrm{Pd}_{3} \mathrm{Ti}\right.$, and $\mathrm{Pd}_{3} \mathrm{Zr}$; space group, $\mathrm{I} 4 / \mathrm{mmm}$, $\mathrm{P}_{3} / \mathrm{mmc} \mathrm{P}_{3} / \mathrm{mmc}$, respectively) were formed in single phases (Fig. 1). The samples were then characterized by
X-ray photoelectron spectroscopy (XPS). Prior to the measurement, the sample ground in air was treated under $\mathrm{H}_{2}$ at $800{ }^{\circ} \mathrm{C}$ for $1 \mathrm{~h}$. The sample was moved into the spectrometer using a portable transfer vessel
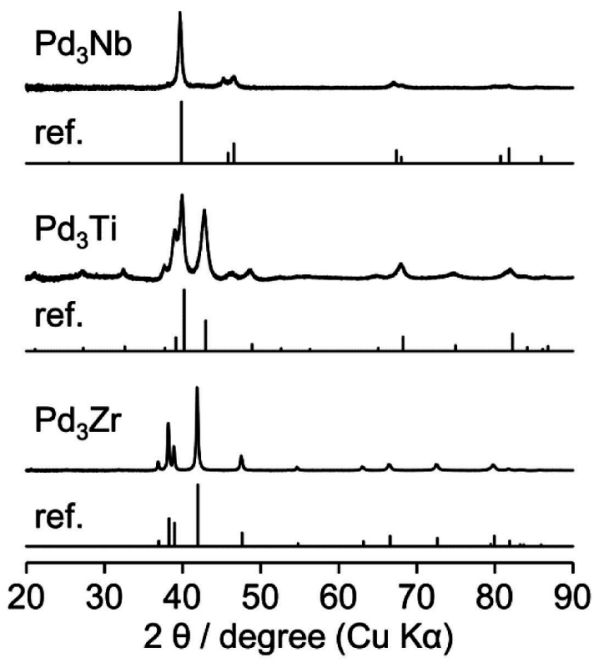

Vertical lines indicate the references: ICDD PDF; \#00-042-1257 (Pd $\left.{ }_{3} \mathrm{Nb}\right), \quad \# 00-035-1361 \quad\left(\mathrm{Pd}_{3} \mathrm{Ti}\right)$, \#01-072-2945 ( $\left.\mathrm{Pd}_{3} \mathrm{Zr}\right)$.

Fig. 1 XRD Patterns of Pd-based Intermetallic Compounds

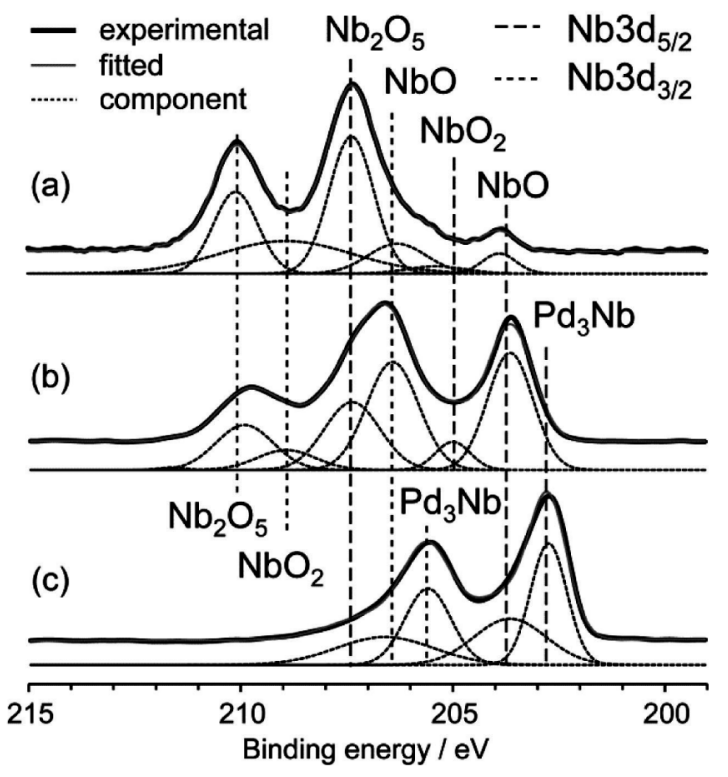

(a) Ground in air, followed by $\mathrm{H}_{2}$ treatment at $800{ }^{\circ} \mathrm{C}$ for $1 \mathrm{~h}$. (b) Ground in $\mathrm{N}_{2}$ in the presence of $<1 \mathrm{ppb} \mathrm{O}_{2}$. (c) The sample (b) after sputtering.

Fig. $\quad 2 \mathrm{Nb} 4 \mathrm{~d}$ XPS of $\mathrm{Pd}_{3} \mathrm{Nb}$ 
without being exposed to air.

Figure 2 shows the $\mathrm{Nb} 3 \mathrm{~d}$ XPS of $\mathrm{Pd}_{3} \mathrm{Nb}$ after various treatments. The sample ground in air (a) shows dominant peaks at $207.4 \mathrm{eV}$ and $210.2 \mathrm{eV}$, assigned to fully oxidized $\mathrm{Nb}^{5+}\left(\mathrm{Nb}_{2} \mathrm{O}_{5}\right)^{7), 8)}$. A small portion of $\mathrm{Nb}^{4+}$ $\left(\mathrm{NbO}_{2} ; 205.0 \mathrm{eV} \text { and } 208.9 \mathrm{eV}\right)^{7)}$ and $\mathrm{Nb}^{2+}(\mathrm{NbO}: 203.8$ $\mathrm{eV}$ and $206.4 \mathrm{eV})^{8)}$ species were also observed. These results demonstrate that the surface $\mathrm{Nb}$ atoms comprising $\mathrm{Pd}_{3} \mathrm{Nb}$ are easily oxidized by air and that the oxidized species cannot be reduced to the metallic state by hydrogen treatment, even at $800{ }^{\circ} \mathrm{C}$. The $\mathrm{Pd}_{3} \mathrm{Nb}$ sample ground in $\mathrm{N}_{2}$ (b) showed dominant peaks assigned to $\mathrm{NbO}$. After sputtering of the oxidized shell (c), new peaks appeared at $203.8 \mathrm{eV}$ and $205.6 \mathrm{eV}$, corresponding to intermetallic $\mathrm{Pb}_{3} \mathrm{Nb}$. No contribution of $\mathrm{Pd}_{3} \mathrm{Nb}$ was observed in the unsputtered sample, indicating that the surface $\mathrm{Nb}$ atoms comprising $\mathrm{Pd}_{3} \mathrm{Nb}$ were completely oxidized, even in the presence of $\mathrm{ppb}$ level oxygen. The corresponding Pd 3d XPS showed that the Pd species was in its metallic state for each sample (data not shown). Based on these results, it can be said that aerobic oxidation of $\mathrm{Pd}_{3} \mathrm{Nb}$ resulted in decomposition to a composite of metallic $\mathrm{Pd}$ and $\mathrm{Nb}$ oxides.

Figure 3 shows the Ti $2 p$ XPS of the $\mathrm{Pd}_{3} \mathrm{Ti}$ intermetallic compound. Similarly to $\mathrm{Pd}_{3} \mathrm{Nb}$, the sample

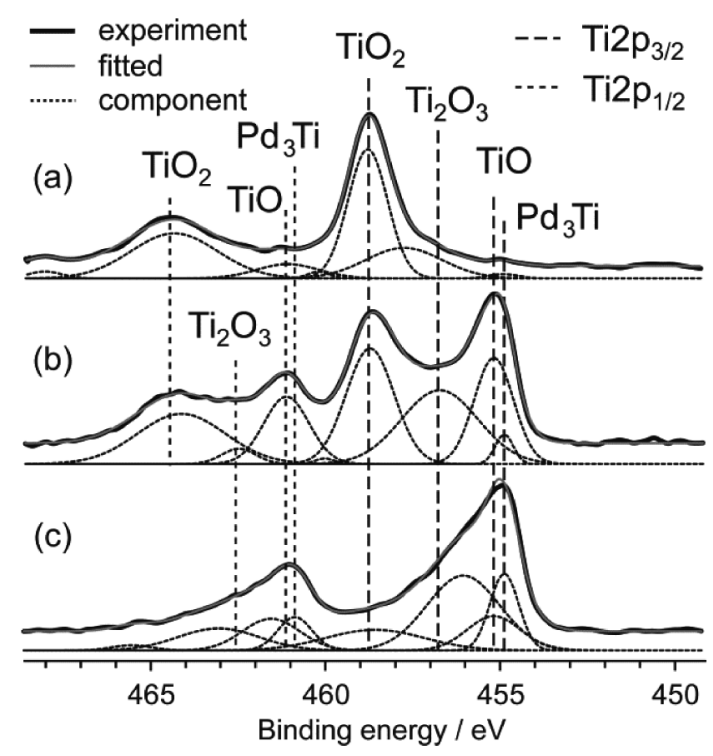

(a) Ground in air, followed by $\mathrm{H}_{2}$ treatment at $800{ }^{\circ} \mathrm{C}$ for 1 h. (b) Ground in $\mathrm{N}_{2}$ in the presence of $<1 \mathrm{ppb} \mathrm{O}_{2}$. (c) The sample (b) after sputtering.

Fig. 3 Ti $2 p$ XPS of $\mathrm{Pd}_{3} \mathrm{Ti}$ ground in air (a) showed dominant peaks assigned to $\mathrm{Ti}^{4+}\left(\mathrm{TiO}_{2}: 458.8 \mathrm{eV} \text { and } 464.5 \mathrm{eV}\right)^{9)}$. The sample ground in $\mathrm{N}_{2}$ (b) showed various peaks corresponding to $\mathrm{TiO}_{2}, \mathrm{Ti}_{2} \mathrm{O}_{3}(456.8 \mathrm{eV} \text { and } 462.5 \mathrm{eV})^{9)}$, $\mathrm{TiO}(455.2$ $\mathrm{eV}$ and $461.1 \mathrm{eV})^{9)}$, and intermetallic $\mathrm{Pd}_{3} \mathrm{Ti}(454.9 \mathrm{eV}$ and $460.8 \mathrm{eV})^{10)}$. Considering that the $\mathrm{Pd}_{3}$ Ti phase has a minor contribution to the whole spectrum, the outermost surface of the sample seems to be covered with the oxide phases. Although the $\mathrm{TiO}_{2}$ phase almost disappeared upon sputtering (c), a significant amount of $\mathrm{Ti}_{2} \mathrm{O}_{3}$ phase remained in addition to the bulk $\mathrm{Pd}_{3} \mathrm{Ti}$ phase. The Pd $3 d$ XPS of $\mathrm{Pd}_{3}$ Ti showed peaks assigned to monometallic $\mathrm{Pd}$ or intermetallic $\mathrm{Pd}_{3} \mathrm{Ti}$ (data not shown). The absence of $\mathrm{Pd}$ oxide phases indicates the oxidative decomposition of the intermetallic $\mathrm{Pd}_{3} \mathrm{Ti}$ into metallic $\mathrm{Pd}$ and Ti oxides, as observed for $\mathrm{Pd}_{3} \mathrm{Nb}$. Thus, for $\mathrm{Pd}_{3} \mathrm{Nb}$ and $\mathrm{Pd}_{3} \mathrm{Ti}$, the surface of the intermetallic compound could not tolerate the ppb level oxygen, demonstrating their remarkably high oxygen sensitivity. Conversely, for $\mathrm{Pd}_{3} \mathrm{Zr}$, a different situation was observed. As shown in Fig. 4, although the sample ground in air showed peaks of $\mathrm{ZrO}_{2}{ }^{11)}$, the sample ground in $\mathrm{N}_{2}$ showed a large contribution of peaks that correspond to the intermetallic $\mathrm{Pd}_{3} \mathrm{Zr}$ phase. No divalent $\mathrm{ZrO}$ species ${ }^{11)}$ were observed in this spectrum. These results indicate that a large part of the $\mathrm{Pd}_{3} \mathrm{Zr}$

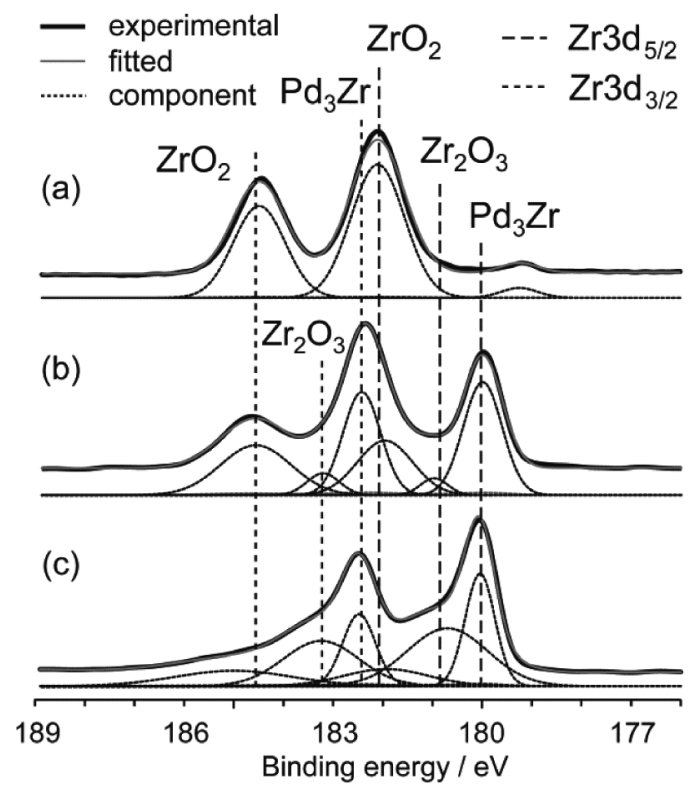

(a) Ground in air, followed by $\mathrm{H}_{2}$ treatment at $800{ }^{\circ} \mathrm{C}$ for $1 \mathrm{~h}$. (b) Ground in $\mathrm{N}_{2}$ in the presence of $<1 \mathrm{ppb} \mathrm{O}_{2}$. (c) The sample (b) after sputtering.

Fig. $4 \quad \mathrm{Zr} 3 \mathrm{~d}$ XPS of $\mathrm{Pd}_{3} \mathrm{Zr}$ 
surface keeps its original state under the $\mathrm{N}_{2}$ atmosphere with a ppb level of oxygen. Thus, the sensitivity to oxygen of the intermetallic compounds depends on the individual intermetallic compounds.

\section{Conclusion}

In summary, we performed XPS analysis of Pd-based intermetallic compounds having early transition metals that were prepared under a ppb-level oxygen atmosphere. The obtained results suggest an intrinsic difficulty in maintaining the original state of the intermetallic compound surface having early transition metals using standard glove box techniques. A surface science technique such as in-situ cutting of an intermetallic crystal under high vacuum would be useful for studying the non-oxidized compounds; however, such a procedure is hardly applicable to commonly used catalytic conditions. Therefore, a much more strict protocol allowing a ppt level oxygen with minimum percolation through the gloves may be required for glove box techniques to be valid for studies dealing with intermetallic compounds having early transition metals as catalysts.

\section{References}

1) Westbrook, J. H., Fleischer, R. L., "Magnetic, Electrical and Optical Properties and Applications of Intermetallic Compounds," Wiley, New York (2000).

2) Komatsu, T., Onda, A., Catal. Surv. Asia, 12, 6 (2008).

3) Furukawa, S., Tamura, A., Ozawa, K., Komatsu, T., Appl. Catal. A: General., 469, 300 (2014).

4) Yamamatsu, S., Yamaguchi, T., Yokota, K., Nagano, O., Chono, M., Aoshima, A., Catal. Surv. Asia, 14, 124 (2010).

5) Furukawa, S., Suga, A., Komatsu, T., Chem. Commun., 50, 3277 (2014).

6) Armbruster, M., Kovnir, K., Behrens, M., Teschner, D., Grin, Y., Schlogl, R., J. Am. Chem. Soc., 132, 14745 (2010).

7) Bahl, M. K., J. Phys. Chem. Solids, 36, 485 (1975).

8) Jha, M., Ramanujachary, K. V., Lofland, S. E., Gupta, G., Ganguli, A. K., Dalton Trans., 40, 7879 (2011).

9) Saied, S. O., Sullivan, J. L., Choudhury, T., Pearce, C. G., Vacuum, 38, 917 (1988).

10) Bzowski, A., Sham, T. K., Phys. Rev. B, 48, 7836 (1993).

11) Degonzalez, O., Garcia, E. A., Surf. Sci., 193, 305 (1988). 